\title{
Generalised Logic Program Transformation Schemas
}

\author{
Halime Büyükyıldız and Pierre Flener \\ Department of Computer Engineering and Information Science \\ Faculty of Engineering, Bilkent University, 06533, Bilkent, Ankara, Turkey \\ pf@cs.bilkent.edu.tr
}

\begin{abstract}
Schema-based logic program transformation has proven to be an effective technique for the optimisation of programs. This paper results from the research that began by investigating the suggestions in 11 to construct a more general database of transformation schemas for optimising logic programs at the declarative level. The proposed transformation schemas fully automate accumulator introduction (also known as descending computational generalisation), tupling generalisation (a special case of structural generalisation), and duality laws (which are extensions to relational programming of the first duality law of the fold operators in functional programming). The schemas are proven correct. A prototype schema-based transformation system is evaluated.
\end{abstract}

\section{Introduction}

Schema-based program construction and synthesis were studied in logic programming 9,10,16,14,23] and in functional programming [20,21]. Using schemas for logic program transformation was first studied in [13] and then extended in [25,18]. Schema-based logic program transformation was also studied in [11,15]. This paper results from the research that began by investigating the suggestions in [11] and extending the ideas in [1] to construct a database of more general transformation schemas for optimising logic programs at the declarative level. For full details of this research, the reader is invited to consult [5].

Throughout this paper, the word program (resp. procedure) is used to mean typed definite program (resp. procedure). An open program is a program where some of the relations appearing in the clause bodies are not appearing in any heads of clauses, and these relations are called undefined (or open) relations. If all the relations appearing in the program are defined, then the program is a closed program. The format of a specification $S_{r}$ of a relation $r$ is:

$$
\forall X: \mathcal{X} . \forall Y: \mathcal{Y} . \mathcal{I}_{r}(X) \Rightarrow\left[r(X, Y) \Leftrightarrow \mathcal{O}_{r}(X, Y)\right]
$$

where $\mathcal{I}_{r}(X)$ denotes the input condition that must be fulfilled before the execution of the procedure, and $\mathcal{O}_{r}(X, Y)$ denotes the output condition that will be fulfilled after the execution.

We now give the definitions of the notions that will be used throughout the paper. All the definitions are given for programs in closed frameworks [12]. A 
framework can be defined simply as a full first-order theory (with identity) with intended model. A closed framework has no parameters and open symbols. Thus, it completely defines an abstract data type (ADT).

Correctness and Equivalence Criteria. We first give correctness and equivalence criteria for programs.

\section{Definition 1 ((Correctness of a Closed Program)).}

Let $P$ be a closed program for a relation $r$ in a closed framework $\mathcal{F}$. We say that $P$ is (totally) correct wrt its specification $S_{r}$ iff, for any ground term $t$ of $\mathcal{X}$ such that $\mathcal{I}_{r}(t)$ holds, we have $P \vdash r(t, u)$ iff $\mathcal{F} \models \mathcal{O}_{r}(t, u)$, for every ground term $u$ of $\mathcal{Y}$. If we replace 'iff' by 'implies' in the condition above, then $P$ is said to be partially correct wrt $S_{r}$, and if we replace 'iff' by 'if', then $P$ is said to be complete wrt $S_{r}$.

This kind of correctness is not entirely satisfactory, for two reasons. First, it defines the correctness of $P$ in terms of the procedures for the relations in its clause bodies, rather than in terms of their specifications. Second, $P$ must be a closed program, even though it might be desirable to discuss the correctness of $P$ without having to fully implement it. So, the abstraction achieved through the introduction (and specification) of the relations in its clause bodies is wasted. This leads us to the notion of steadfastness (also known as parametric correctness) 129 .

\section{Definition 2 ((Steadfastness of an Open Program)).}

In a closed framework $\mathcal{F}$, let:

- $P$ be an open program for a relation $r$

$-q_{1}, \ldots, q_{m}$ be all the undefined relation names appearing in $P$

$-S_{1}, \ldots, S_{m}$ be the specifications of $q_{1}, \ldots, q_{m}$.

We say that $P$ is steadfast wrt its specification $S_{r}$ in $\left\{S_{1}, \ldots, S_{m}\right\}$ iff the (closed) program $P \cup P_{S}$ is correct wrt $S_{r}$, where $P_{S}$ is any closed program such that:

- $P_{S}$ is correct wrt each specification $S_{j}(1 \leq j \leq m)$

- $P_{S}$ contains no occurrences of the relations defined in $P$.

For program equivalence, now, we do not require the two programs to have the same models, because this would not make much sense in some program transformation settings, where the transformed program features relations that are not in the initially given program. That is why our program equivalence criterion establishes equivalence wrt the specification of a common relation (usually the root of their call-hierarchies).

\section{Definition 3 ((Equivalence of Two Open Programs)).}

In a closed framework $\mathcal{F}$, let $P$ and $Q$ be two open programs for a relation $r$. Let $S_{1}, \ldots, S_{m}$ be the specifications of $p_{1}, \ldots, p_{m}$, which are all the undefined relation names appearing in $P$, and let $S_{1}^{\prime}, \ldots, S_{t}^{\prime}$ be the specifications of 
$q_{1}, \ldots, q_{t}$, which are all the undefined relation names appearing in $Q$. We say that $\left\langle P,\left\{S_{1}, \ldots, S_{m}\right\}\right\rangle$ is equivalent to $\left\langle Q,\left\{S_{1}^{\prime}, \ldots, S_{t}^{\prime}\right\}\right\rangle$ wrt the specification $S_{r}$ (or simply that $P$ is equivalent to $Q$ wrt $S_{r}$ ) when $P$ is steadfast wrt $S_{r}$ in $\left\{S_{1}, \ldots, S_{m}\right\}$ and $Q$ is steadfast wrt $S_{r}$ in $\left\{S_{1}^{\prime}, \ldots, S_{t}^{\prime}\right\}$. Since the 'is equivalent to' relation is symmetric, we also say that $P$ and $Q$ are equivalent wrt $S_{r}$.

In program transformation settings, there sometimes are conditions that have to be satisfied by some parts of the initial and/or transformed program in order to have a transformed program that is equivalent to the initially given program wrt the specification of the top-level relation. Hence the following definition.

\section{Definition 4 ((Conditional Equivalence of Two Open Programs)).}

In a closed framework $\mathcal{F}$, let $P$ and $Q$ be two open programs for a relation $r$. We say that $P$ is equivalent to $Q$ wrt the specification $S_{r}$ under conditions $C$ iff $P$ is equivalent to $Q$ wrt $S_{r}$ whenever $C$ holds.

Program Schemas and Schema Patterns. The notion of program schema was also used in [910 11 13 16 14 15 625 , but here we have an additional component, which makes our definition [12] of program schemas different.

\section{Definition 5 ((Program Schemas)).}

In a closed framework $\mathcal{F}$, a program schema for a relation $r$ is a pair $\langle T, C\rangle$, where $T$ is an open program for $r$, called the template, and $C$ is a set of specifications of the open relations of $T$, in terms of each other and in terms of the input/output conditions of the closed relations of $T$. The specifications in $C$, called the steadfastness constraints, are such that, in $\mathcal{F}, T$ is steadfast wrt its specification $S_{r}$ in $C$.

Sometimes, a series of schemas are quite similar, in the sense that they only differ in the number of arguments of some relation, or in the number of calls to some relation, etc. For this purpose, rather than having a proliferation of similar schemas, we introduce the notion of schema pattern (compare with [6]).

\section{Definition 6 ((Schema Patterns)).}

A schema pattern is a program schema where term, conjunct, and disjunct ellipses are allowed in the template and in the steadfastness constraints.

For instance, $T X_{1}, \ldots, T X_{t}$ is a term ellipsis, and $\bigwedge_{i=1}^{t} r\left(T X_{i}, T Y_{i}\right)$ is a conjunct ellipsis. Our schemas are more general than those in [11] in the sense that we now allow such ellipses.

Schema-based Program Transformation. In schema-based transformation, transformation techniques are pre-compiled at the schema-level.

\section{Definition 7 ((Transformation Schemas)).}

A transformation schema is a 5 -tuple $\left\langle S_{1}, S_{2}, A, O_{12}, O_{21}\right\rangle$, where $S_{1}$ and $S_{2}$ are program schemas (or schema patterns), $A$ is a set of applicability conditions, 
which ensure the equivalence of the templates of $S_{1}$ and $S_{2}$ wrt the specification of the top-level relation, and $\mathrm{O}_{12}$ (resp. $\mathrm{O}_{21}$ ) is a set of optimisability conditions, which ensure further optimisability of the output program schema (or schema pattern) $S_{2}$ (resp. $S_{1}$ ).

If a transformation schema embodies some generalisation technique, then it is called a generalisation schema. The problem generalisation techniques that are used in this paper are explained in detail in [9]. Using these techniques for synthesising and/or transforming a program in a schema-based fashion was first proposed in 910, and then extended in 11. The generalisation methods that we pre-compile in our transformation schemas are tupling generalisation, which is a special case of structural generalisation where the structure of some parameter is generalised, and descending generalisation, which is a special case of computational generalisation where the general state of computation is generalised in terms of what remains to be done. If a transformation schema embodies a duality law, then it is called a duality schema.

In the remainder of this paper, we first give two divide-and-conquer schema patterns in Section 2. We then explain in detail how automation of program transformation is achieved by tupling and descending generalisation, in Sections 3 and 4. In Section 5, we explain the duality schemas. In Section 6, we discuss, by using the results of performance tests, the effects of the optimisability conditions in the transformation schemas. Before we conclude, the prototype transformation system, which was developed to test the practicality of the ideas explained in this paper, is presented in Section 7 .

\section{Divide-and-Conquer Programs}

The schema patterns in this section abstract sub-families of divide-and-conquer (DC) programs. They are here restricted to binary relations with $X$ as the induction parameter and $Y$ as the result parameter, to reflect the schema patterns that can be handled by the prototype transformation system explained in Section 7 . Another restriction in the schema patterns is that when $X$ is non-minimal, then $X$ is decomposed into $h=1$ head $H X$ and $t>0$ tails $T X_{1}, \ldots, T X_{t}$, so that $Y$ is composed from 1 head $H Y$ (which is the result of processing $H X$ ) and $t$ tails $T Y_{1}, \ldots, T Y_{t}$ (which are the results of recursively calling the relation with $T X_{1}, \ldots, T X_{t}$, respectively) by $p$-fix composition (i.e., $Y$ is composed by putting its head $H Y$ between its tails $T Y_{p-1}$ and $\left.T Y_{p}\right)$.

These schema patterns are called $D C L R$ and $D C R L$ (the reason for these names will be explained soon). Template $D C L R$ (resp. $D C R L$ ) below is the template of the $D C L R$ (resp. $D C R L$ ) schema pattern. In these patterns, minimal, solve, etc., denote place-holders for relation symbols. During the particularisation of a schema pattern to a schema, all these place-holders are renamed, because otherwise all divide-and-conquer programs would have the same relation symbols. Indeed, since a template is an open program, the idea is to obtain 
concrete programs from the template by adding programs for the open relations, such that these programs satisfy the steadfastness constraints. The steadfastness constraints corresponding to these DC templates (i.e., the specifications of their open relations) are the same, since these templates have the same open relations. Such constraints are shown in 12 in this volume.

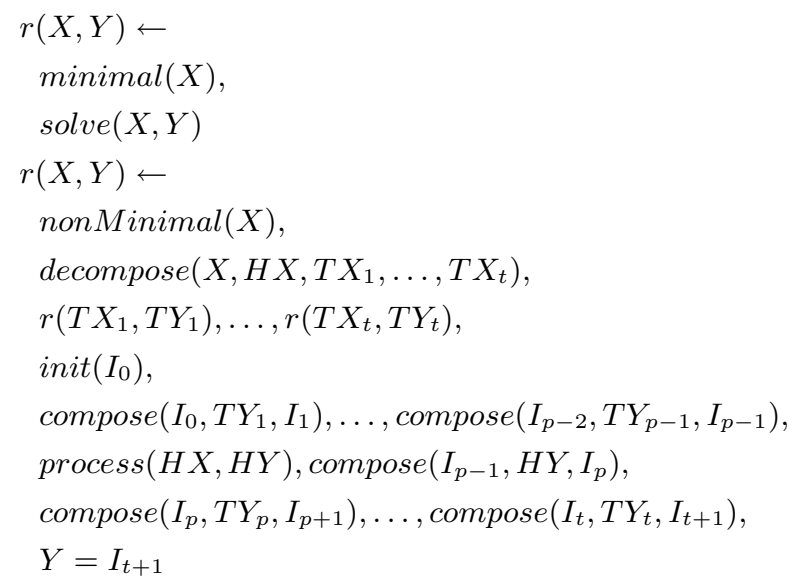

Template $D C L R$

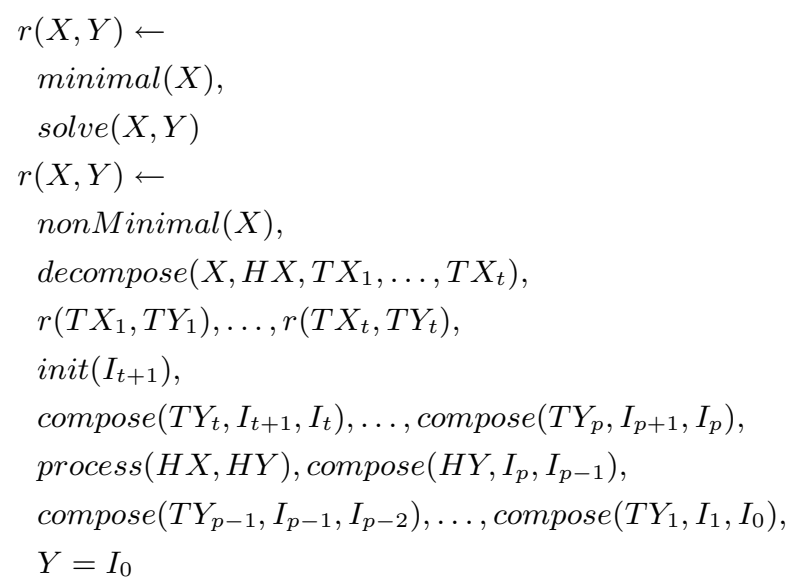

\section{Template $D C R L$}

We can now explain the underlying idea why we have two different schema patterns for DC, and why we call them $D C L R$ and $D C R L$. If we denote the functional version of the compose relation with $\oplus$, then the composition of $Y$ in template $D C L R$ by left-to-right $(L R)$ composition ordering can be written as:

$$
\left.Y=\left(\left(\left(\left(\left(e \oplus T Y_{1}\right) \oplus \ldots\right) \oplus T Y_{p-1}\right) \oplus H Y\right) \oplus T Y_{p}\right) \oplus \ldots\right) \oplus T Y_{t}
$$


where $e$ is the (unique) term for which init holds. Similarly, the composition of $Y$ in $D C R L$ by right-to-left $(R L)$ composition ordering can be written as:

$$
Y=T Y_{1} \oplus\left(\ldots \oplus\left(T Y_{p-1} \oplus\left(H Y \oplus\left(T Y_{p} \oplus\left(\ldots \oplus\left(T Y_{t} \oplus e\right)\right)\right)\right)\right)\right)
$$

Throughout the paper, we use the infix_flat problem, whose DC programs are given in the example below.

Example 1. The specification of infix_flat is:

infix_flat $(B, F)$ iff list $F$ is the infix representation of binary tree $B$

where infix representation means the list representation of the infix traversal of the tree. Program 1 (resp. Program 2) below is the program for the infix_flat/2 problem that is a (partially evaluated) instance of the $D C L R$ (resp. DCRL) schema pattern, for $t=p=2$. Note the line-by-line correspondence between the program computations and the templates.

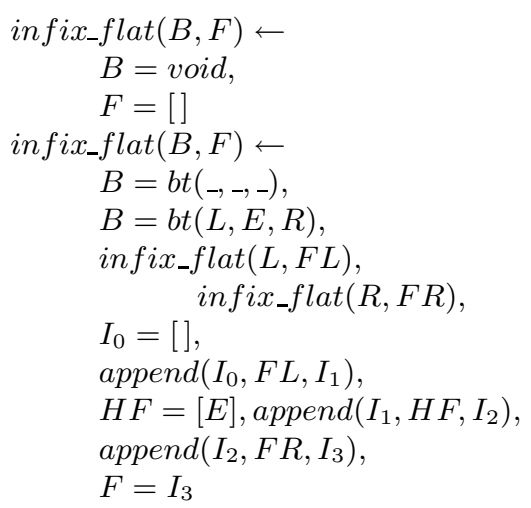

Program 1

$$
\begin{aligned}
& \text { infix_flat }(B, F) \leftarrow \\
& B=\text { void }, \\
& F=[] \\
& \text { infix_flat }(B, F) \leftarrow \\
& B=b t\left({ }_{-},-,\right), \\
& B=b t(L, E, R), \\
& \text { infix_flat }(L, F L), \\
& \quad \text { infix_flat }(R, F R), \\
& I_{3}=[], \\
& \text { append }\left(F R, I_{3}, I_{2}\right), \\
& \text { HF }=[E], \text { append }\left(H F, I_{2}, I_{1}\right), \\
& \text { append }\left(F L, I_{1}, I_{0}\right), \\
& F=I_{0}
\end{aligned}
$$

\section{Program 2}

\section{Program Transformation by Tupling Generalisation}

If a program for a relation $r$, which has the specification $S_{r}$ of Section 1 is given as an instance of $D C L R$ (or $D C R L$ ), then the specification of the tuplinggeneralised problem of $r$, namely $S_{r_{-} \text {tupling }}$, is:

$$
\begin{array}{r}
\forall X s: \text { list of } \mathcal{X} . \forall Y: \mathcal{Y} . \quad\left(\forall X: \mathcal{X} . X \in X s \Rightarrow \mathcal{I}_{r}(X)\right) \Rightarrow \\
\left(r _ { - } \text { tupling } ( X s , Y ) \Leftrightarrow ( X s = [ ] \wedge Y = e ) \vee \left(X s=\left[X_{1}, X_{2}, \ldots, X_{n}\right]\right.\right. \\
\left.\left.\wedge \bigwedge_{i=1}^{n} \mathcal{O}_{r}\left(X_{i}, Y_{i}\right) \wedge I_{1}=Y_{1} \wedge \bigwedge_{i=2}^{n} \mathcal{O}_{c}\left(I_{i-1}, Y_{i}, I_{i}\right) \wedge Y=I_{n}\right)\right)
\end{array}
$$

where $\mathcal{O}_{c}$ is the output condition of compose, and $e$ is the (unique) term for which init holds. 
The tupling generalisation schemas (one for each $D C$ schema pattern) are:

$T G_{1}:\left\langle D C L R, T G, A_{t_{1}}, O_{t_{1} 12}, O_{t_{1} 21}\right\rangle$ where

$A_{t_{1}}:$ - compose is associative

- compose has $e$ as the left and right identity element

- $\forall X: \mathcal{X} . \mathcal{I}_{r}(X) \wedge \operatorname{minimal}(X) \Rightarrow \mathcal{O}_{r}(X, e)$

- $\forall X: \mathcal{X} . \quad \mathcal{I}_{r}(X) \Rightarrow[\neg \operatorname{minimal}(X) \Leftrightarrow \operatorname{nonMinimal}(X)]$

$O_{t_{1} 12}:$ partial evaluation of the conjunction process $(H X, H Y)$, compose $(H Y, T Y, Y)$

results in the introduction of a non-recursively defined relation

$O_{t_{1} 21}$ : partial evaluation of the conjunction

process $(H X, H Y)$, compose $\left(I_{p-1}, H Y, I_{p}\right)$

results in the introduction of a non-recursively defined relation

$T G_{2}:\left\langle D C R L, T G, A_{t_{2}}, O_{t_{2} 12}, O_{t_{2} 21}\right\rangle$ where

$A_{t_{2}}$ : - compose is associative

- compose has $e$ as the left and right identity element

- $\forall X: \mathcal{X} . \mathcal{I}_{r}(X) \wedge \operatorname{minimal}(X) \Rightarrow \mathcal{O}_{r}(X, e)$

- $\forall X: \mathcal{X} . \mathcal{I}_{r}(X) \Rightarrow[\neg \operatorname{minimal}(X) \Leftrightarrow \operatorname{nonMinimal}(X)]$

$O_{t_{2} 12}:$ partial evaluation of the conjunction process $(H X, H Y)$, compose $(H Y, T Y, Y)$

results in the introduction of a non-recursively defined relation

$\mathrm{O}_{t_{2} 21}$ : partial evaluation of the conjunction process $(H X, H Y)$, compose $\left(H Y, I_{p}, I_{p-1}\right)$

results in the introduction of a non-recursively defined relation

where the template of the common schema pattern $T G$ is given on the next page.

Note that, in the TG template, all the open relations of $D C L R$ (or $D C R L$ ) appear, but no new relations. This crucial observation enables the once-and-forall verification of the conditional equivalence of the two templates in the $T G_{i}$ transformation schemas wrt $S_{r}$ under $A_{t_{i}}$, which verification is thus independent of the actual specifications of the open relations (i.e., the steadfastness constraints) 4 .

The applicability conditions of $T G_{1}$ (resp. $T G_{2}$ ) ensure the equivalence of the $D C L R$ (resp. DCRL) and $T G$ programs for a given problem. The optimisability conditions ensure that the output programs of these generalisation schemas can be made more efficient than the input programs. Indeed, the optimisability conditions, together with some of the applicability conditions, check whether the compose calls in the $T G$ template can be eliminated. For instance, the conjunction solve $(X, H Y)$, compose $(H Y, T Y, Y)$ in the second clause of $r_{-} t u p l i n g$ can be simplified to $Y=A$, if relation $r$ maps the minimal form of $X$ into $e$, and if $e$ is also the right identity element of compose. This is already checked by the second and third applicability conditions of $T G_{1}$ and $T G_{2}$. Also, in the third and fourth clauses of r_tupling, the conjunction process $(H X, H Y)$, compose $(H Y, T Y, Y)$ can be partially evaluated, resulting in the disappearance of that call to compose, and thus in a merging of the compose loop into the $r$ loop in the DCLR (or $D C R L)$ template, if the optimisability condition $O_{t_{1} 12}$ (or $O_{t_{2} 12}$ ) holds.

Let us illustrate tupling generalisation by applying the $T G_{i}$ generalisation schemas on the infix_flat problem. 


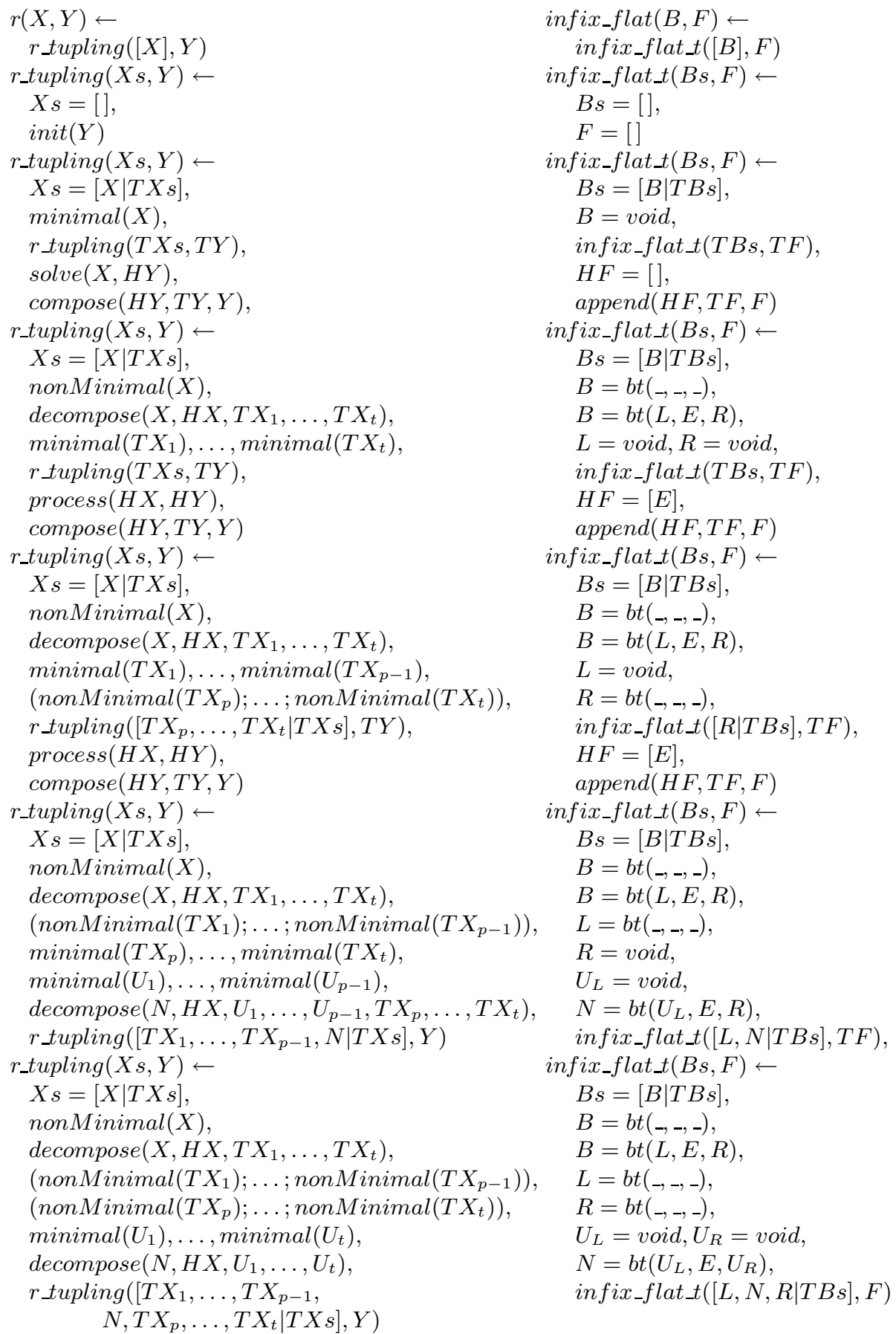

Template $T G$ and Program 3 
Example 2. The specification of the infix_flat problem, and its DCLR and $D C R L$ programs, are in Example 1 in Section 2. The infix_flat problem can be tupling-generalised using the $T G_{i}$ transformation schemas above, since the infix_flat programs have open relations that satisfy the applicability and optimisability conditions of these schemas. So, the specification of the tuplinggeneralised problem of infix_flat is:

infix_flat_t $(B s, F)$ iff $F$ is the concatenation of the infix representations of the elements in the binary tree list $B s$.

Program 3 on the previous page is the tupling-generalised program for in fix_flat as an instance of $T G$, for $t=p=2$.

Although the tupling generalisation schemas are constructed to tuplinggeneralise DC programs (i.e., to transform DC programs into TG programs), these schemas can also be used in the reverse direction, such that they transform TG programs into DC programs, provided the optimisability conditions for the corresponding $D C$ schema pattern are satisfied; note that applicability conditions work in both directions.

\section{Program Transformation by Descending Generalisation}

Descending generalisation [9] can also be called the accumulation strategy (as in functional programming [2, and in logic programming [17]), since it introduces an accumulator parameter and progressively extends it to the final result. Descending generalisation can also be seen as transformation towards difference structure manipulation, since any form of difference structures can be created by descending generalisation, and not just difference-lists.

Four descending generalisation schemas (two for each DC schema pattern) are given. Since the applicability conditions of each descending generalisation schema are different, the process of choosing the appropriate generalisation schema for the input DC program is done only by checking the applicability and optimisability conditions, and the eureka (i.e., the specification of the generalised problem) then comes for free.

The reason why we call the descendingly generalised (DG) schema patterns ' $D G L R$ ' and ' $D G R L$ ' is similar to the reason why we call the divide-and-conquer schema patterns $D C L R$ and $D C R L$, respectively. In descending generalisation, the composition ordering for extending the accumulator parameter in the template $D G L R$ (resp. $D G R L)$ is from left-to-right (LR) (resp. right-to-left (RL)).

The first two descending generalisation schemas are:

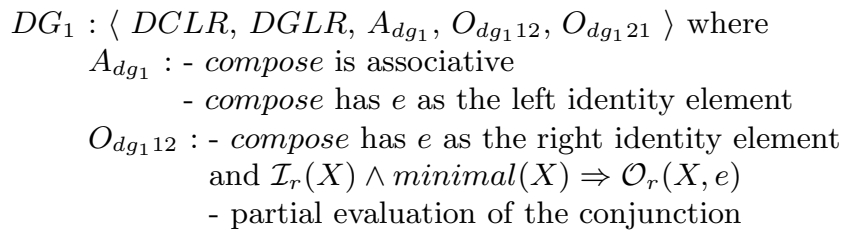




$$
\begin{aligned}
& \operatorname{process}(H X, H Y) \text {, compose }\left(A_{p-1}, H Y, A_{p}\right) \\
& \text { results in the introduction of a non-recursively defined relation } \\
O_{d g_{1} 21}: & - \text { partial evaluation of the conjunction } \\
& \text { process }(H X, H Y) \text {, compose }\left(I_{p-1}, H Y, I_{p}\right) \\
& \text { results in the introduction of a non-recursively defined relation }
\end{aligned}
$$

$D G_{4}:\left\langle D C R L, D G L R, A_{d g_{4}}, O_{d g_{4} 12}, O_{d g_{4} 21}\right\rangle$ where

$A_{d g_{4}}$ : - compose is associative

- compose has $e$ as the left and right identity element

$O_{d g_{4} 12}:-\mathcal{I}_{r}(X) \wedge \operatorname{minimal}(X) \Rightarrow \mathcal{O}_{r}(X, e)$

- partial evaluation of the conjunction

process $(H X, H Y)$, compose $\left(A_{p-1}, H Y, A_{p}\right)$

results in the introduction of a non-recursively defined relation

$O_{d g_{4} 21}$ : - partial evaluation of the conjunction process $(H X, H Y)$, compose $\left(H Y, I_{p}, I_{p-1}\right)$

results in the introduction of a non-recursively defined relation

where $e$ is the (unique) term for which init holds. These schemas have the same formal specification (i.e., eureka) for the relation $r_{-}$descending $g_{1}$ of the schema pattern $D G L R$, namely:

$$
\begin{aligned}
\forall X: \mathcal{X} . \forall Y, A: \mathcal{Y} . \mathcal{I}_{r}(X) & \Rightarrow \\
\quad\left[r_{\text {_descending }}(X, Y, A)\right. & \left.\Leftrightarrow \exists S: \mathcal{Y} . \mathcal{O}_{r}(X, S) \wedge \mathcal{O}_{c}(A, S, Y)\right]
\end{aligned}
$$

where $\mathcal{O}_{c}$ is the output condition of compose. The template of the common schema pattern $D G L R$ of $D G_{1}$ and $D G_{4}$ is:

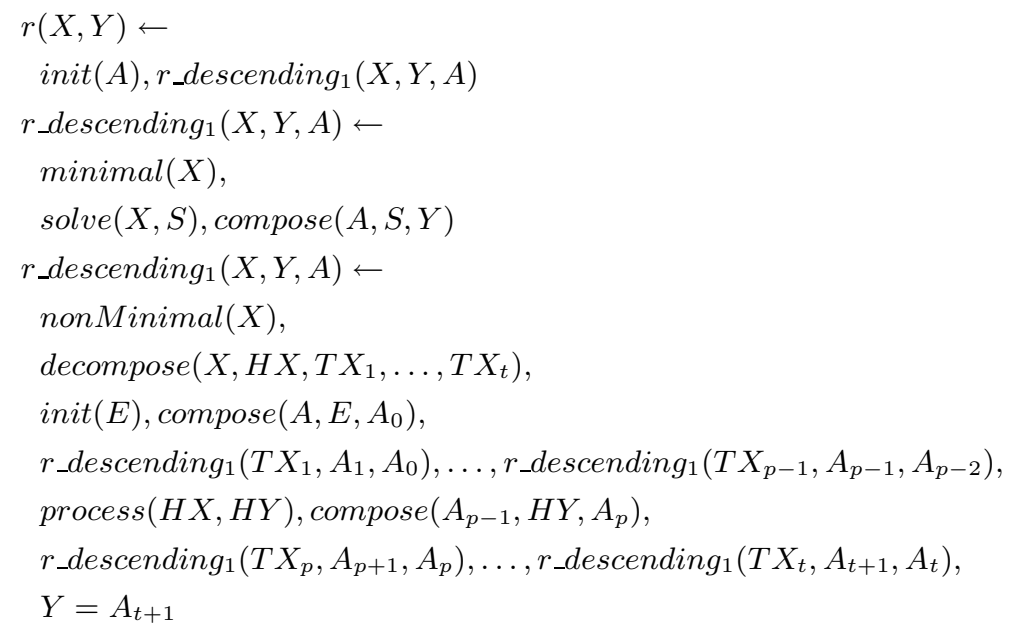

\section{Template $D G L R$}

Note that, in the DGLR template, all the open relations of $D C L R$ (or $D C R L$ ) appear, but no new relations. The applicability and optimisability conditions of these two generalisation schemas differ, since the composition ordering is changed from RL to $\mathrm{LR}$ in $D G_{4}$.

We now illustrate descending generalisation on our infix_flat problem. 
Example 3. The specification of a program for the LR descendingly generalised version of infix_flat is:

infix_flat_descending $g_{1}(B, F, A)$ iff list $F$ is the concatenation of list $A$ and the infix representation of binary tree $B$.

Program 4 is the program for infix $f$ flat as an instance of $D G L R$, for $t=p=2$.

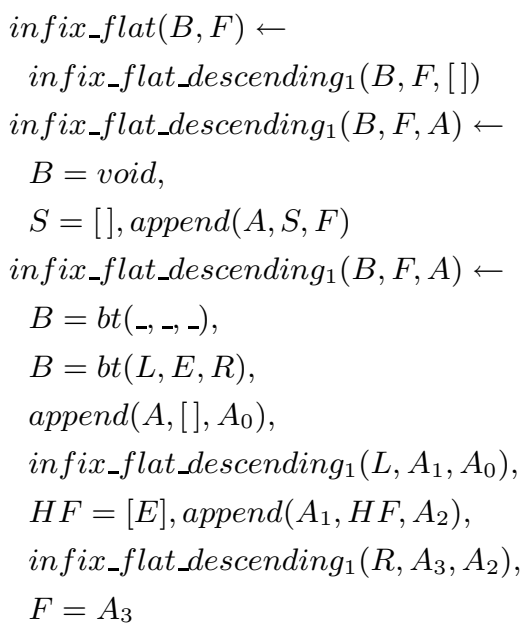

\section{Program 4}

Since the applicability conditions of $D G_{1}$ (resp. $D G_{4}$ ) are satisfied for the input $D C L R$ (resp. DCRL) infix_flat program, the descendingly generalised infix_flat program can be Program 4. However, for this problem, descending generalisation of the infix_flat programs with the $D G$ transformation schemas above should not be done, since the optimisability conditions of $D G_{1}$ (resp. $D G_{4}$ ) are not satisfied by the open relations of infix_flat. Indeed, in the nonminimal case of infix_flat_descending , $_{1}$, partial evaluation of the conjunction $H F=[E]$, append $\left(A_{1}, H F, A_{2}\right)$ does not result in the introduction of a nonrecursively defined relation, because of properties of append (actually, due to the inductive definition of lists). Moreover, the induction parameter of append, which is here the accumulator parameter, increases in length each time append is called in the non-minimal case, which shows that this program is not a good choice as a descendingly generalised program for this problem. So, the optimisability conditions are really useful to prevent non-optimising transformations.

The other two descending generalisation schemas are:

$D G_{2}:\left\langle D C L R, D G R L, A_{d g_{2}}, O_{d g_{2} 12}, O_{d g_{2} 21}\right\rangle$ where

$A_{d g_{2}}$ : - compose is associative

- compose has $e$ as the left and right identity element

$O_{d g_{2} 12}:-\mathcal{I}_{r}(X) \wedge \operatorname{minimal}(X) \Rightarrow \mathcal{O}_{r}(X, e)$

- partial evaluation of the conjunction 


$$
\begin{aligned}
& \operatorname{process}(H X, H Y), \text { compose }\left(H Y, A_{p}, A_{p-1}\right) \\
& \text { results in the introduction of a non-recursively defined relation } \\
O_{d g_{2} 21}: & \text { - partial evaluation of the conjunction } \\
& \text { process }(H X, H Y) \text {, compose }\left(I_{p-1}, H Y, I_{p}\right) \\
& \text { results in the introduction of a non-recursively defined relation }
\end{aligned}
$$

$D G_{3}:\left\langle D C R L, D G R L, A_{d g_{3}}, O_{d g_{3} 12}, O_{d g_{3} 21}\right\rangle$ where

$A_{d g_{3}}:$ - compose is associative

- compose has $e$ as the right identity element

$O_{d g_{3} 12}:-$ compose has $e$ as the left identity element and $\mathcal{I}_{r}(X) \wedge \operatorname{minimal}(X) \Rightarrow \mathcal{O}_{r}(X, e)$

- partial evaluation of the conjunction process $(H X, H Y)$, compose $\left(H Y, A_{p}, A_{p-1}\right)$

results in the introduction of a non-recursively defined relation

$O_{d g_{3} 21}$ : - partial evaluation of the conjunction

process $(H X, H Y)$, compose $\left(H Y, I_{p}, I_{p-1}\right)$

results in the introduction of a non-recursively defined relation

where $e$ is the (unique) term for which init holds. These schemas have the same formal specification (i.e., eureka) for the relation $r_{-}$descending $g_{2}$ of the schema pattern $D G R L$, namely:

$$
\begin{aligned}
\forall X: \mathcal{X} . \forall Y, A: \mathcal{Y} . \mathcal{I}_{r}(X) & \Rightarrow \\
{\left[r_{\text {_ddescending }}(X, Y, A)\right.} & \left.\Leftrightarrow \exists S: \mathcal{Y} . \mathcal{O}_{r}(X, S) \wedge \mathcal{O}_{c}(S, A, Y)\right]
\end{aligned}
$$

where $\mathcal{O}_{c}$ is the output condition of compose. Note the reversal of the roles of $A$ and $S$ compared to the specification of $r_{-}$descending $g_{1}$ above. The template of the common schema pattern $D G R L$ of $D G_{2}$ and $D G_{3}$ is:

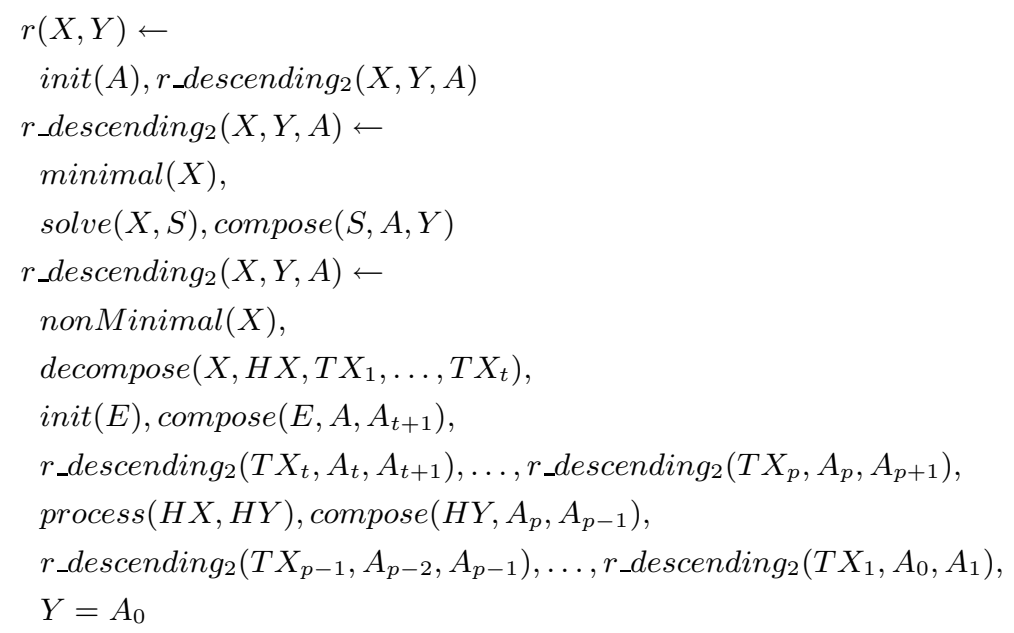

\section{Template $D G R L$}

Note that, in the $D G R L$ template, all the open relations of $D C L R$ (or DCRL) appear, but no new relations. The applicability and optimisability conditions of these two generalisation schemas differ, since the composition ordering is changed from LR to RL in $D G_{2}$. 
Example 4. The specification of a program for the RL descendingly generalised version of infix_flat is:

infix_flat_descending $g_{2}(B, F, A)$ iff list $F$ is the concatenation of the infix representation of binary tree $B$ and list $A$.

Program 5 is the program for infix flat as an instance of $D G R L$, for $t=p=2$.

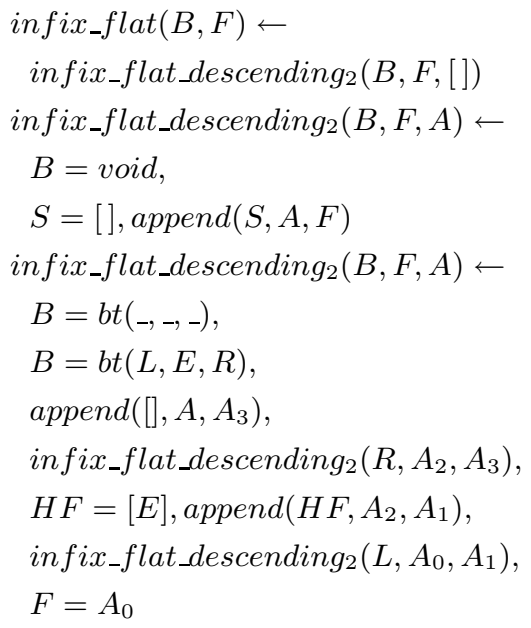

\section{Program 5}

Since both the applicability conditions and the optimisability conditions of $D G_{2}$ (resp. $D G_{3}$ ) are satisfied for the input $D C L R$ (resp. DCRL) infix_flat program, descending generalisation of the in fix_flat programs results in Program 5. Partial evaluation of the conjunction $H F=[E]$, append $\left(H F, A_{2}, A_{1}\right)$ in the nonminimal case of infix_flat_descending 2 then results in $A_{1}=\left[E \mid A_{2}\right]$. Similarly, partial evaluation of the conjunction $S=$ [], append $(S, A, F)$ in the minimal case results in $F=A$. Altogether, this amounts to the elimination of append.

Although the descending generalisation schemas are constructed to descendingly generalise DC programs, these schemas can also be used to transform DG programs into DC programs, provided the optimisability conditions for the corresponding DC schema pattern are satisfied. It is thus possible that we have Program 4 for the infix_flat problem, and that we want to transform it into a more efficient program; then the DC programs are good candidates, if we have the descending generalisation schemas above.

\section{Program Transformation Using Duality Laws}

In Section 2, while we discussed the composition ordering in the DC program schemas, the reader who is familiar with functional programming has noticed 
the similarities with the fold operators in functional programming. A detailed explanation of the fold operators and their laws can be found in 3 . Here, we only give the definitions of the fold operators, and their first law. The foldr operator can be defined as follows:

$$
\text { foldr }(\oplus) a\left[x_{1}, x_{2}, \ldots, x_{n}\right]=x_{1} \oplus\left(x_{2} \oplus\left(\ldots\left(x_{n} \oplus a\right) \ldots\right)\right)
$$

where $\oplus$ is a variable that is bound to a function of two arguments. Similarly, the foldl operator can be defined as follows:

$$
\text { foldl }(\oplus) a\left[x_{1}, x_{2}, \ldots, x_{n}\right]=\left(\ldots\left(\left(a \oplus x_{1}\right) \oplus x_{2}\right) \ldots\right) \oplus x_{n}
$$

Thus, equation (1) in Section 2], which illustrates the composition of $Y$ in the $D C L R$ template, can be rewritten using foldl:

$$
Y=f o l d l(\oplus) e\left[T Y_{1}, \ldots, T Y_{p-1}, H Y, T Y_{p}, \ldots, T Y_{t}\right]
$$

Similarly, the foldr operator can be used to rewrite equation (2), which illustrates the composition of $Y$ in the $D C R L$ template:

$$
Y=\operatorname{foldr}(\oplus) e\left[T Y_{1}, \ldots, T Y_{p-1}, H Y, T Y_{p}, \ldots, T Y_{t}\right]
$$

The first three laws of the fold operators are called duality theorems. The first duality theorem states that:

$$
\text { foldr }(\oplus) \text { a } x s=\text { foldl }(\oplus) \text { a } x s
$$

if $\oplus$ is associative and has (left/right) identity element $a$, and $x s$ is a finite list.

By adding optimisability conditions, we can now devise a transformation schema based on this first duality theorem (compare with [16]):

$D_{d c}:\left\langle D C L R, D C R L, A_{d d c}, O_{d d c 12}, O_{d d c 21}\right\rangle$ where

$A_{d d c}$ : - compose is associative

- compose has $e$ as the left and right identity element

$O_{d d c 12}$ : - partial evaluation of the conjunction process $(H X, H Y)$, compose $\left(H Y, I_{p}, I_{p-1}\right)$

results in the introduction of a non-recursively defined relation

$O_{d d c 21}$ : - partial evaluation of the conjunction process $(H X, H Y)$, compose $\left(I_{p-1}, H Y, I_{p}\right)$

results in the introduction of a non-recursively defined relation

where $e$ is the (unique) term for which init holds, where the schema patterns $D C L R$ and DCRL are given in Section 2 and where $A_{d d c}$ comes from the constraints of the first duality theorem. The optimisability conditions check whether the compose operator can be eliminated in the output program.

Similarly, it is possible to give a duality schema between the $D G$ schema patterns:

$D_{d g}:\left\langle D G L R, D G R L, A_{d d g}, O_{d d g 12}, O_{d d g 21}\right\rangle$ where

$A_{d d g}:$ - compose is associative

- compose has $e$ as the left and right identity element 


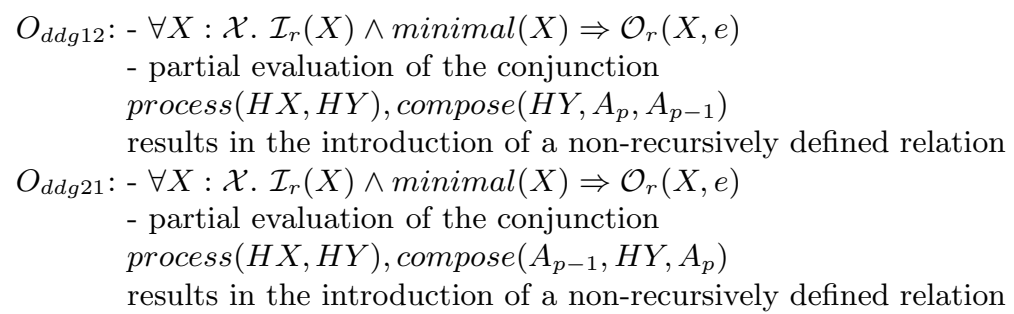

where $e$ is the (unique) term for which init holds, and where the schema patterns $D G L R$ and $D G R L$ are given in Section 4 .

\section{Evaluation of the Transformation Schemas}

We evaluate the transformation schemas using performance tests done on partially evaluated input and output programs of each transformation schema. However, the reader may find this evaluation a little bit dubious, since the transformation schemas in this paper are only dealing with the declarative features of programs. This evaluation is made because we think that these performance tests will help us see what our theoretical results amount to when tested practically, although in an environment with procedural side-effects. The programs are executed using Mercury 0.6 (for an overview of Mercury, please refer to [22]) on a SPARCstation 4. Since the programs are really short, the procedures were called 500 or 1000 times to achieve meaningful timing results. In Table 1, the results of the performance tests for five selected relations are shown, where each column heading represents the schema pattern to which the program written for the relation of that row belongs. (Of course, quicksort is not really a relation: we just mean to indicate that some partitioning is used as decompose for the sort relation.) The timing results are normalised wrt the DCLR column.

\begin{tabular}{|c|r|r|r|r|r|}
\hline relations & DCLR & DCRL & TG & DGLR & DGRL \\
\hline Prefix flat & 1.00 & 0.92 & 0.23 & 11.88 & 0.15 \\
\hline Infix flat & 1.00 & 0.49 & 0.02 & 7.78 & 0.05 \\
\hline Postfix flat & 1.00 & 0.69 & 0.14 & 5.48 & 0.09 \\
\hline reverse & 1.00 & 1.00 & 0.04 & 1.01 & 0.01 \\
\hline quicksort & 1.00 & 0.85 & 0.72 & 6.02 & 0.56 \\
\hline
\end{tabular}

Table 1. Performance test results

The reason why we chose the relations above is that for all the five considered schema patterns programs can be written for these relations.

Let us first compare the $D C L R$ and $D C R L$ schema patterns. For reverse, the $D C L R$ and $D C R L$ programs are the same, since they are singly recursive, and their compose relation is append, which is associative. For the binary tree flat relations and for quicksort, the $D C R L$ programs are much better than the 
$D C L R$ programs, because of properties of relations like append (which is the compose relation in all these programs), which are the main reason for achieving the optimisations of the $D C R L$ programs for the relations above. Hence, if the input programs for the binary tree flat relations and for the quicksort problem to the duality schema are instances of the $D C L R$ schema pattern, then a duality transformation will be performed, resulting in $D C R L$ programs for these relations, since both the applicability and the optimisability conditions are satisfied by these programs. If the $D C R L$ programs for the relations above are input to the duality schema, then the duality transformation will not be performed, since the optimisability conditions are not satisfied by append, which is the compose relation of the $D C R L$ programs. Of course, there may exist some other relations where the duality transformation of their $D C R L$ programs into the $D C L R$ programs will provide an efficiency gain. Unfortunately, we could not find a meaningful relation of this category.

The next step in evaluating the transformation schemas is to compare the generalised programs of these example relations. If we look at Table 1, the most obvious observation is that the $D G R L$ programs for all these relations are very efficient programs. However, tupling generalisation seems to be the second best as a generalisation choice, and it must even be the first choice for relations like infix flat, where the composition place of the head in the result parameter is in the middle, and where the minimal and non Minimal checks can be performed in minimum time. Although a similar situation occurs for quicksort, its TG program is not quite as efficient as its $D G R L$ program. This is mainly because of partition, which is the decompose relation of quicksort, being a costly operation, although we eliminated most of the partition calls by putting extra minimality checks into the TG template. Since append, which is the compose relation in all the programs, cannot be eliminated in the resulting $D G L R$ programs, the $D G L R$ programs for these relations have the worst timing results. The reason for their bad performance is that the percentages of the total running times of the $D G L R$ programs used by append are much higher than the percentages of the total running times of the $D C L R$ and $D C R L$ programs used by append for these relations. The reason for the increase in the percentages is that the length of the accumulator, which is the input parameter to append in the DGLR programs, is larger than the length of the input parameter of append in the DCLR and $D C R L$ programs, since the partial result has to be repeatedly input to the compose relation in descending generalisation.

A transformation should be performed only if it really results in a program that is more efficient than the input program. So, for instance, the descending generalisation of the input $D C L R$ program for infix flat resulting in the $D G L R$ program must not be done, even though the applicability conditions are satisfied. This is the main reason for the existence of the optimisability conditions in the schemas.

In some of the cases, using generalisation schemas to transform input programs that are already generalised programs into DC programs can produce an efficiency gain. For example, if the $D G L R$ program for any of the flat relations 
is the input program to descending generalisation (namely $D G_{1}$ or $D G_{4}$ ), then a de-generalisation will be performed resulting in the $D C L R$ (or $D C R L$ ) program, which is more efficient than the input $D G L R$ program. However, with the current optimisability conditions, if the input program for any of the relations above to generalisation is a $D G R L$ program, then the generalisation schemas are still applied in the reverse direction, resulting in a $D C R L$ program, which means that the de-generalisation will result in a program that is less efficient than the input program. This makes us think of even more accurate ways of defining the optimisability conditions, namely as actual optimisation conditions, such that the transformation will always result in a better program than the input program. However, more performance analyses and complexity analyses are needed to derive such conditions.

\section{A Prototype Transformation System}

TRANSYS is a prototypical implementation of the schema-based program transformation approach summarised in this paper. TRANSYS is a fully automatic program transformation system and was developed to be integrated with a schema-guided program development environment. Therefore, the input program to TRANSYs is assumed to be developed by a synthesiser using the database of schema patterns known to TRANSYS. The schema pattern of which the input program is an instance is thus a priori known, and so are the renamings of the open relation symbols, the particularisations of the schema variables such as $t$ and $p$, as well as the "closing" programs defining these open relations of the template. In other words, no matching between the input program and the templates of the transformation schemas has to be performed, unlike in [6 25]. Given an input program, TRANSys outputs (what it believes to be) the best programs that are more efficient than the input program: this is done by collecting the leaves of the tree rooted in that input program and where child nodes are developed when both the applicability and the optimisability conditions of a transformation schema hold. All the transformation schemas and the schema patterns, which are the input (or output) schema patterns of these transformation schemas, given in [5] (i.e., a superset of the schemas given in this paper), are available in the database of the system.

TranSys has been developed in SICStus Prolog 3. Since TranSys is a prototype system, for some parts of the system, instead of implementing them ourselves, we reused and integrated other systems:

- For verifying the applicability conditions and some of the optimisability conditions, PTTP is integrated into the system. The Prolog Technology Theorem Prover (PTTP) was developed by M. Stickel (for a detailed explanation of PTTP, the reader can refer to 24]). PTTP is an implementation of the model elimination theorem proving procedure for the full first-order predicate calculus. TRANSys uses the version of PTTP that is written in Prolog and that compiles clauses into Prolog. 
- For verifying the other optimisability conditions, and for applying these optimisations to the output programs of the transformation schemas, we integrated Mixtus 0.3.6. Mixtus was developed by D. Sahlin (for a detailed explanation of Mixtus, the reader can refer to [19]). Mixtus is an automatic partial evaluator for full Prolog. Given a Prolog program and a query, it will produce a new program specialised for all instances of that query. The partial evaluator is guaranteed to terminate for all input programs and queries.

For a detailed explanation of the TRANSYS system, the reader is invited to consult [5].

\section{Conclusions and Future Work}

This paper results from the research that began by investigating the suggestions in 11. The contributions of this research are:

- pre-compilation of more general generalisation schemas (tupling and descending) than those in [11, which were restricted to sub-families of divideand-conquer programs;

- discovery of the duality schemas;

- discovery of the optimisability conditions;

- validation of the correctness of the transformation schemas, based on the notions of correctness of a program, steadfastness of a program in a set of specifications, and equivalence of two programs (the correctness proofs of the transformation schemas given in this paper and in [5] can be found in [4]; another approach to validation of transformation schemas can be found in [18);

- development of a prototype transformation system;

- validation of the effectiveness of the transformation schemas by performance tests.

This research opens future work directions, such as:

- extension to normal programs and open frameworks;

- consideration of other program schemas (or schema patterns);

- extension of the schema pattern language so as to express even more general program families;

- representation of the loop merging strategy as a transformation schema;

- search for other transformation schemas;

- identification of optimisation conditions that always ensure improved performance (or complexity) of the output program wrt the input program;

- validation of the effectiveness of the transformation schemas by automated complexity analysis (using GAIA [7] and/or CASLOG []). 
Acknowledgments. We wish to thank the anonymous reviewers of the previous versions of this paper as well as the participants of the LOPSTR'97 workshop for their valuable comments and suggestions, especially Yves Deville (UC Louvain, Belgium). We also gratefully acknowledge the feedback of the students of the second author's Automated Software Engineering course at Bilkent, especially Necip Fazıl Ayan, Brahim Hnich, Ayşe Pınar Saygın, Tuba Yavuz, and Cemal Yilmaz.

\section{References}

1. T. Batu. Schema-Guided Transformations of Logic Algorithms. Senior Project Report, Bilkent University, Department of Computer Science, 1996.

2. R.S. Bird. The promotion and accumulation strategies in transformational programming. ACM Transactions on Programming Languages and Systems 6(4):487504, 1984.

3. R.S. Bird and P. Wadler. Introduction to Functional Programming. Prentice Hall, 1988.

4. H. Büyükyıldız and P. Flener. Correctness Proofs of Transformation Schemas. Technical Report BU-CEIS-9713. Bilkent University, Department of Computer Science, 1997.

5. H. Büyükyıldız. Schema-based Logic Program Transformation. M.Sc. Thesis, Technical Report BU-CEIS-9714. Bilkent University, Department of Computer Science, 1997.

6. E. Chasseur and Y. Deville. Logic program schemas, semi-unification, and constraints. In: N.E. Fuchs (ed), Proc. of LOPSTR'97 (this volume).

7. A. Cortesi, B. Le Charlier, and S. Rossi. Specification-based automatic verification of Prolog programs. In: J. Gallagher (ed), Proc. of LOPSTR'96, pp. 38-57. LNCS 1207. Springer-Verlag, 1997.

8. S.K. Debray and N.W. Lin. Cost analysis of logic programs. ACM TOPLAS 15(5):826-875, 1993.

9. Y. Deville. Logic Programming: Systematic Program Development. AddisonWesley, 1990.

10. Y. Deville and J. Burnay. Generalization and program schemata: A step towards computer-aided construction of logic programs. In: E.L. Lusk and R.A. Overbeek (eds), Proc. of NACLP'89, pp. 409-425. The MIT Press, 1989.

11. P. Flener and Y. Deville. Logic program transformation through generalization schemata. In: M. Proietti (ed), Proc. of LOPSTR'95, pp. 171-173. LNCS 1048. Springer-Verlag, 1996.

12. P. Flener, K.-K. Lau, and M. Ornaghi. On correct program schemas. In: N.E. Fuchs (ed), Proc. of LOPSTR'97 (this volume).

13. N.E. Fuchs and M.P.J. Fromherz. Schema-based transformation of logic programs. In: T. Clement and K.-K. Lau (eds), Proc. of LOPSTR'91, pp. 111-125. Springer Verlag, 1992.

14. T.S. Gegg-Harrison. Representing logic program schemata in $\lambda$ Prolog. In: L. Sterling (ed), Proc. of ICLP'95, pp. 467-481. The MIT Press, 1995.

15. T.S. Gegg-Harrison. Extensible logic program schemata. In: J. Gallagher (ed), Proc. of LOPSTR'96, pp. 256-274. LNCS 1207. Springer-Verlag, 1997.

16. A. Hamfelt and J. Fischer Nilsson. Declarative logic programming with primitive recursion relations on lists. In: L. Sterling (ed), Proc of JICSLP'96. The MIT Press. 
17. A. Pettorossi and M. Proietti. Transformation of logic programs: foundations and techniques. Journal of Logic Programming 19(20):261-320, 1994.

18. J. Richardson and N.E. Fuchs. Development of correct transformation schemata for Prolog programs. In: N.E. Fuchs (ed), Proc. of LOPSTR'97 (this volume).

19. D. Sahlin. An Automatic Partial Evaluator of Full Prolog. Ph.D. Thesis, Swedish Institute of Computer Science, 1991.

20. D.R. Smith. Top-down synthesis of divide-and-conquer algorithms. Artificial Intelligence 27(1):43-96, 1985.

21. D.R. Smith. KIDS: A semiautomatic program development system. IEEE Transactions on Software Engineering 16(9):1024-1043, 1990.

22. Z. Somogyi, F. Henderson, and T. Conway. Mercury: An efficient purely declarative logic programming language. In: Proc. of the Australian Computer Science Conference, pp. 499-512, 1995.

23. L.S. Sterling and M. Kirschenbaum. Applying techniques to skeletons. In: J.M. Jacquet (ed), Constructing Logic Programs, pp. 127-140, John Wiley, 1993.

24. M.E. Stickel. A Prolog technology theorem prover: A new exposition and implementation in Prolog. Theoretical Computer Science 104:109-128, 1992.

25. W.W. Vasconcelos and N.E. Fuchs. An opportunistic approach for logic program analysis and optimisation using enhanced schema-based transformations. In: M. Proietti (ed), Proc. of LOPSTR'95, pp. 174-188. LNCS 1048. Springer-Verlag, 1996. 